\title{
Estudio de Pertinencia de los Cuidados Paliativos en Ecuador.
} \section{Study of the Relevance of Palliative Care in Ecuador:}

\section{Mariana Vallejo Martínez ${ }^{1 *}$ iD, Nancy Lino1, Rina Quinto².}

1. Servicio de Dolor y cuidados Paliativos del Instituto Oncológico Nacional "Dr. Juan Tanca Marengo" Solca, Guayaquil-Ecuador.

*Correspondencia:

mcvallejom@hotmail.es

Teléfono [593] 043718700

Conflicto de intereses: Los autores declaran no tener conflictos de intereses.

Fondos: Ver la página 251

Recibido: 3 Junio 2016

Aceptado: 20 Junio 2017

Publicado: 30 Diciembre 2017

\section{Membrete bibliográfico:}

Vallejo M, Lino N, Quinto R. Estudio de pertinencia de los Cuidados Paliativos en Ecuador. Rev. Oncol. Ecu 2017;27(3):238-252.

DOI: https://doi.org/10.33821/241

Copyright Vallejo, et al. Este artículo es distribuido bajo los términos de Creative Commons Attribution License, el cual permite el uso y redistribución citando la fuente y al autor original.
2. Servicio de Estadística del Instituto Oncológico Nacional "Dr. Juan Tanca Marengo" Solca, Guayaquil-Ecuador.

\section{Resumen}

Introducción: El objetivo del presente escrito es proporcionar una guía de análisis de la pertinencia en los cuidados paliativos como una necesidad imperiosa, se analiza la tasa de mortalidad con la relación de la necesidad de este servicio en la provincia del Guayas.

Métodos: El presente estudio descriptivo cuali y cuantitativo; se consultó el archivo estadístico del Instituto Oncológico Nacional "Dr. Juan Tanca Marengo" Solca-Guayaquil y el INEC. Se consultó la incidencia de cáncer del período 2008-2012 de las ciudades de Guayaquil, Quito, Cuenca, Loja y provincia de Manabí. Se utilizó un estudio de mercado en 100 usuarios del área médica para la implementación de la especialidad en cuidados paliativos, diseñada por la Asociación Latinoamericana de Cuidados Paliativos (ALCP).

Resultados: en Guayaquil la tasa de incidencia de cáncer en hombres es de 142.1 y mujeres 165.5, mientras que en Quito en hombres es 228.1 por cada 100 mil habitantes, y 230.1 en mujeres por cada 100 mil habitantes. La tasa de crecimiento por cáncer es del $4 \%$ anual. En la encuesta 100 trabajadores de la salud, $100 \%$ consideró muy necesaria la especialidad de cuidados paliativos, el 93 $\%$ desconoce si se oferta la especialidad, $100 \%$ respondió que las universidades deberían ofertar esta especialidad, de los 9 temas que se deben abordar en la especialidad de cuidados paliativos, el tratamiento del dolor tema fue considerado el más relevante por el $100 \%$ de los encuestados. La Universidad Católica de Santiago de Guayaquil fue elegida por el $86 \%$ de los encuestados y el centro escogido para el desarrollo de la especialización fue Solca, en el $100 \%$ de los encuestados.

Conclusión: Se infiere que es sumamente importante contar con programas de posgrado para iniciar la formación de especialistas en Cuidados Paliativos debido a que es un personal profesional estratégico, para elevar los estándares de calidad de vida de la población. Ecuador no registra atención profesional paliativista y las Facultades de Ciencias Médicas en Ecuador no registran programas académicos.

Palabras Claves: CUIDADOS PALIATIVOS, EDUCACIÓN DE POSGRADO, PROGRAMAS, NEOPLASIAS. 


\section{Abstract}

Introduction: The aim of this paper is to provide a guide to the analysis of the relevance of palliative care as an urgent need, the mortality rate is analyzed with the relation of the need for this service in the province of Guayas.

Methods: The present qualitative and quantitative descriptive study; We consulted the statistical file of the National Oncological Institute "Dr. Juan Tanca Marengo "Solca-Guayaquil and the INEC. The cancer incidence of the 2008-2012 period was consulted in the cities of Guayaquil, Quito, Cuenca, Loja and the province of Manabí. A market study was used in 100 users of the medical area for the implementation of the specialty in palliative care, designed by the Latin American Association of Palliative Care (ALCP).

Results: in Guayaquil the incidence rate of cancer in men is 142.1 and women 165.5 , while in Quito in men it is 228.1 per 100 thousand inhabitants, and 230.1 in women per 100 thousand inhabitants. The cancer growth rate is $4 \%$ per year. In the survey 100 health workers, $100 \%$ considered the specialty of palliative care very necessary, $93 \%$ did not know if the specialty was offered, $100 \%$ answered that universities should offer this specialty, of the 9 subjects that should be addressed in The specialty of palliative care, the treatment of pain topic was considered the most relevant by $100 \%$ of the respondents. The Catholic University of Santiago de Guayaquil was chosen by $86 \%$ of the respondents and the center chosen for the development of the specialization was Solca, in $100 \%$ of the respondents.

Conclusion: It is inferred that it is extremely important to have postgraduate programs to start the training of specialists in Palliative Care because it is a strategic professional staff, to raise the standards of quality of life of the population. Ecuador does not register professional palliative care and the Faculties of Medical Sciences in Ecuador do not register academic programs.

Keywords: PALLIATIVE CARE, POSTGRADUATE EDUCATION, PROGRAMS, NEOPLASMS.

DOI: $10.33821 / 241$

\section{Introducción}

La revolución diagnóstica y terapéutica de los últimos 50 años junto a la mejoría progresiva de las condiciones sociales y económicas han logrado aumentar la sobrevida de la población de poco más de 60 años a casi 80 , incluido en países en desarrollo. Ello ha traído consigo un aumento considerable del número de pacientes con enfermedades crónicas incurables, incluidos los pacientes con cáncer metastásico, accidentes vasculares, demencias, las insuficiencias hepáticas, cardíacas, respiratorias, renales y enfermos de SIDA. Son estos los pacientes que despertaron las raíces compasivas de la medicina a mediados de los sesenta en el siglo pasado en Inglaterra, donde la preocupación central se puso en el control de síntomas, control total del dolor [1] y mejoría de la calidad de vida, incluyendo los aspectos familiares, sociales, psicológicos y espirituales de los pacientes que están por morir y sus familiares [2].

Si se considera que fallecen 56 millones de personas al año, el $85 \%$ de ellas en países en vías de desarrollo como Ecuador, se asume que cada muerte también afecta a por lo menos otras 5 personas en términos de relación informal y asistencia al duelo de la familia y los 
amigos. Esto es una estimación bastante modesta especialmente en aquellos países en vías de desarrollo, donde la tasa de natalidad todavía es alta. El total de personas que se encuentra afectada cada año en el mundo por problemas relacionados con la atención de los pacientes moribundos es alrededor de 300 millones es decir, el $5 \%$ de la población mundial. Esto hace que la calidad de la atención al final de la vida sea uno de los problemas de salud pública global más importante que se enfrentan hoy en el mundo [3].

En muchos países desarrollados hay un reconocimiento más profundo que en el nuestro sobre las necesidades de mejorar el cuidado de los pacientes incurables en el final de su vida. La cultura materialista de la sociedad y los avances tecnológicos en medicina han oscurecido la necesidad del desarrollo de una medicina más humana y compasiva para el enfermo que está por morir y su familia.

La Organización Mundial de la Salud (OMS) define los cuidados paliativos (CP) como «el enfoque que mejora la calidad de vida de pacientes y familias que se enfrentan a los problemas asociados con enfermedades amenazantes para la vida, a través de la prevención y el alivio del sufrimiento, por medio de la identificación temprana y la impecable evaluación y tratamiento del dolor y otros problemas físicos, psicosociales y espirituales».

Considera que el equipo socio-sanitario debe aproximarse a los enfermos y a sus familiares con el objetivo de responder a sus necesidades, y enumera las siguientes características de los cuidados paliativos:

- Proporcionan el alivio del dolor y de otros síntomas que producen sufrimiento.

- Promocionan la vida y consideran la muerte como un proceso natural.

- No se proponen acelerar el proceso de morir ni retrasarlo.

- Integran los aspectos psicosociales y espirituales en los cuidados del paciente.

- Tienen en cuenta el soporte y los recursos necesarios para ayudar a los pacientes a vivir de la manera más activa posible hasta su muerte.

- Ofrecen apoyo a los familiares y a los allegados durante la enfermedad y el duelo.

- Mejoran la calidad de vida del paciente.

- Se aplican desde las fases tempranas de la enfermedad junto con otras terapias dirigidas a prolongar la vida (como la quimioterapia, radioterapia, etc.).

- Incluyen también las investigaciones necesarias para comprender mejor y manejar situaciones clínicas complejas.

Esta concepción de los CP reconoce que las personas con enfermedades distintas al cáncer, que sean irreversibles, progresivas y con una fase terminal, también pueden beneficiarse de su aplicación. Pueden ser, por ejemplo, los pacientes con enfermedad pulmonar obstructiva crónica (EPOC), demencia, insuficiencia cardíaca, insuficiencia renal o hepática avanzadas o enfermedades neurológicas (como ictus, parkinson, esclerosis múltiple o esclerosis lateral amiotrófica, entre otras) [4].

Como se muestra en la figura 1, los CP deberían comenzar en las fases tempranas del diagnóstico de una enfermedad que amenaza la vida, simultáneamente con los tratamientos curativos. De la misma forma, incluso en las fases finales de la enfermedad, en las que el tratamiento es predominantemente paliativo, puede existir un espacio para el intento destinado a las medidas curativas. 
Figura 1. Entorno de los Cuidados Paliativos.

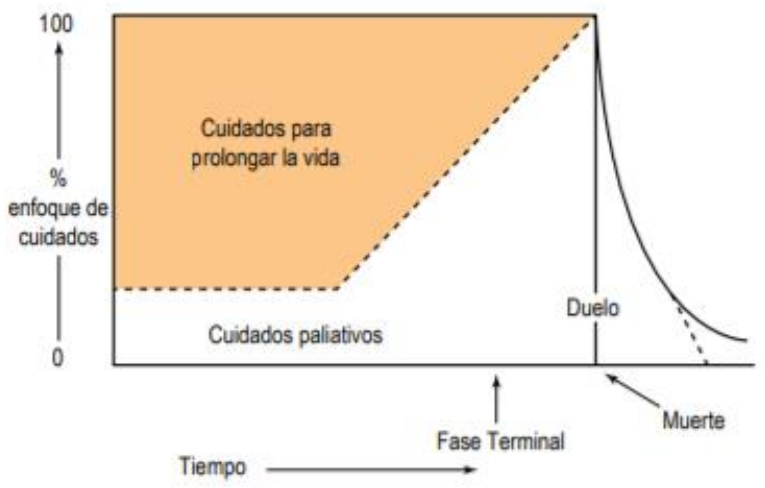

Adaptada de la Universidad de California con permiso de Steven Z. Pantilat, MD, FACP.

Por otro lado, incluye la atención del duelo. Así, la transición de los cuidados curativos a paliativos es a menudo gradual, y debe basarse en las necesidades individuales de la persona más que en un plazo concreto de supervivencia esperada. En este contexto la ley orgánica de la Salud de la República del Ecuador manifiesta en el artículo 10 que "Quienes forman parte del Sistema Nacional de Salud aplicarán las políticas, programas y normas de atención integral y de calidad, que incluyen acciones de promoción, prevención, recuperación, rehabilitación y cuidados paliativos de la salud individual y colectiva." Y el Acuerdo Ministerial 101 publicado en el Registro Oficial No. 415 de 29 de marzo de 2011, se dispuso organizar, en el marco del Modelo de Atención Integral del Ministerio de Salud Pública, la conformación y funcionamiento de servicios de cuidados paliativos integrales con enfoque intercultural, que contribuyan a garantizar los derechos de los pacientes en etapa terminal a aliviar el dolor y el sufrimiento y a abordar los aspectos físicos, emocionales, sociales y espirituales, incluyendo a los familiares, cuando sea necesario; [5] y el 17 de noviembre de 2014, la Directora Nacional de Políticas y Modelamiento del Sistema Nacional de Salud, solicitó la elaboración del Acuerdo Ministerial aprobó en el artículo 1 "la publicación del documento denominado "Plan Nacional de Cuidados Paliativos 2015 - 2017", el mismo que es de aplicación y observancia obligatoria en todos los establecimientos que conforman el Sistema Nacional de Salud, según el artículo 2.

Entre los objetivos generales de los $\mathrm{CP}$, se destacan la promoción del bienestar y la mejora de la calidad de vida, y se considera necesario lo siguiente:

1. Una información y comunicación adecuadas, que aseguren al enfermo y a su familia que serán escuchados y que obtendrán unas respuestas claras y sinceras. Además se les proporcionará el apoyo emocional que precisen, de manera que puedan expresar sus emociones y participar en la toma de decisiones con respecto a su vida y al proceso de morir con apego a las leyes vigentes.

2. La atención del dolor y otros síntomas físicos, así como de las necesidades emocionales, sociales y espirituales, sin olvidar aquellos aspectos prácticos del cuidado de los enfermos y de sus familiares. 
3. Una continuidad asistencial asegurada a lo largo de su evolución, estableciendo mecanismos de coordinación entre todos los ámbitos asistenciales y servicios implicados.

El Ministerio de Salud, con la participación de la Red Pública Integral de Salud (RPIS) y la Red Privada Complementaria (RPC), el Ministerio de Salud Pública, elaboró la Política Nacional de Cuidados Paliativos, estos son el apoyo y soporte médico que se da para mejorar la calidad de vida y tratar el dolor en los pacientes con enfermedades crónicas avanzadas o con pronóstico de vida limitado y de sus familias. Para esta construcción se tomaron en cuenta cinco líneas estratégicas:

1. Desarrollo de un marco jurídico.

2. Implementación de las prestaciones de cuidados paliativos.

3. Disponibilidad y acceso a medicamentos.

4. Participación de la sociedad.

5. Investigación.

El trabajo que se realizó, se vio fortalecido por la participación de un experto en la implementación de Políticas Públicas de Cuidados Paliativos, el médico español José Espinosa Rojas, Coordinador del Centro Colaborador de la OMS para Programas Públicos de Cuidados Paliativos del Instituto Catalán de Oncología; quien permaneció en el país del 5 al 9 de agosto colaborando con un equipo ecuatoriano con autoformación en los cuidados paliativos.

Con esta política se motivó la acción conjunta de los profesionales de cuidados paliativos, tanto del sector público como del privado y conformar una comisión interinstitucional, y adoptar una Guía práctica clínica y una Guía de paliativos para el ciudadano que facilite a todos los profesionales de la salud, cuidadores y pacientes la atención de cuidados paliativos con toma de decisiones adecuadas y respaldadas científicamente [6].

Los problemas actuales que existen a nivel mundial, obligan al ser humano a pensar de manera universal y proponer soluciones desde una visión multidimensional e interdisciplinaria, de forma que pueda articular, organizar y contextualizar los nuevos conocimientos para que solo entonces adquieran significado y relevancia. Según los fundamentos teóricos planteados por Edgar Morín [7] la irrupción del paradigma dela complejidad y la propuesta del pensamiento complejo, en el paisaje científico contemporáneo, ha provocado un replantamiento de los fundamentos mismos de la racionalidad occidental. Su repercusión en el campo pedagógico se ha evidenciado en la medida que pone entre paréntesis los conceptos, conocimientos t aprendizaje al cuestionar sus fundamentos epistémicos. Ha planteado además, la necesidad de reformar el pensamiento $y$, al mismo tiempo reformar las instituciones educativas, con el fin de promover una educación acorde con las necesidades de la sociedad contemporánea y esta reforma de pensamiento según Morín, coincide con el concepto de medicina paliativa.

El Ecuador apuesta al desarrollo del talento humano y la calidad educativa como principios claves para transitar de una economía primaria a una nueva basada en el conocimiento. En este marco, la universidad ecuatoriana enfrenta el gran desafío de ser fuente generadora del cambio por encontrarse en un sitial privilegiado, al poder incidir en las personas y 
contribuir a esa reforma del pensamiento que se requiere para consolidar una sociedad del buen vivir, (Larrea, 2014) [8].

Por otro lado, el Consejo de Evaluación, Acreditación y Aseguramiento de la Calidad de la Educación Superior (CEAACES), propone un modelo de evaluación del entorno de aprendizaje de carreras presenciales y semipresenciales de las universidades y escuelas politécnicas, en el que uno de sus criterios es el de pertinencia, a través del cual "se evalúa que la carrera o programa académico, responda a las expectativas y necesidades de la sociedad, a la planificación nacional, a la prospectiva de desarrollo científico, humanístico y tecnológico mundial y a la diversidad cultural, como sustento para la elaboración del perfil profesional" [9]. Los nuevos horizontes de formación del talento humano de la nación deberán centrarse en sus capacidades cognitivas para la creación y re construcción del conocimiento en contextos de investigación e innovación. Para ello la educación superior deberá proporcionar ambientes de aprendizaje con claras dinámicas de organización del conocimiento y los saberes, que produzcan espacios sociales y epistemológicos para interpretar los problemas de la ciencia y la realidad, orientados a la implicación con su transformación, en el marco de una formación ciudadana e intercultural. Es importante considerar que el criterio de pertinencia definido por el CEAACES para la evaluación de carreras es consecuente con el propuesto por el CES para la formulación del rediseño curricular o diseño de nuevas carreras.

El modelo del CEAACES determina dos subcriterios que componen el criterio pertinencia: "Contexto" que exige que la propuesta académica esté respaldada por procesos de planificación, gestión, y sea coherente con la información general presentada, pertinencia y análisis de la evolución prospectiva de la carrera; y, "Profesión" que demanda que la formación académica responda al perfil profesional [10]. En este contexto y considerando la normativa establecida por el CES y las exigencias de calidad del CEAACES, el presente estudio de pertinencia de los cuidados paliativos que presenta la UCSG y SOLCA se basa en las siguientes variables: objetivos del Plan Nacional del Buen Vivir (PNBV) [11], y de la política Nacional de los cuidados paliativos del MSP, líneas de acción para la transformación de la matriz productiva; oferta y demanda educativa a nivel nacional; expectativa estudiantil por la carrera del área de cuidados paliativos que oferta la UCSG, demanda de la necesidad país. El objetivo del presente estudio es proporcionar una guía de análisis de la pertinencia en los cuidados paliativos como una necesidad imperiosa, se analiza la tasa de mortalidad con la relación de la necesidad de este servicio en la provincia del Guayas.

\section{Materiales y Métodos}

En el presente trabajo se realizó una investigación descriptiva en términos cualitativos y cuantitativos; se utilizó para el efecto fuentes primarias del archivo estadístico del Instituto Oncológico Nacional "Dr. Juan Tanca Marengo" Solca-Guayaquil y el INEC [12].

Se consultó la incidencia de cáncer del período 2008-2012 de las ciudades de Guayaquil, Quito, Cuenca, Loja y provincia de Manabí, las cuales representan el 40 \% de la población 
del país, esto publicado en el libro Cancer Incidence in Five Continents Vol. XI de la IARC [14].

Se utilizó un estudio de mercado para la implementación de la especialidad en cuidados paliativos, además una herramienta diseñada por la Asociación Latinoamericana de Cuidados Paliativos (ALCP) [13] para calcular la necesidad de cuidados paliativos en la región que utiliza las siguientes variables:

a) Análisis de la tasa de mortalidad por cáncer proporcionada por el INEC, Anuario de Estadísticas vitales 2016

b) Análisis de la tasa de mortalidad por ENT proporcionada por el INEC, Anuario de Estadísticas vitales 2016

c) Análisis de porcentaje de cuidadores necesarios para el manejo paliativo

d) Expectativa de los profesionales de la salud por carreras del área de cuidados paliativos que se realizó en base a estudios de mercado 2017.

La herramienta para conocer la necesidad estimada de cuidados paliativos en los pacientes con enfermedades crónicas, diseñada por R.Wenk y publicada en el Link de la Asociación Latinoamericana de cuidados paliativos (ALCP), consideran los fallecimientos por enfermedades cardiovasculares, diabetes, digestivas, cáncer, enfermedades pulmonares crónicas, y otras enfermedades no transmisibles (ENT). Se utilizaron los fallecimientos registrados en la provincia del Guayas de acuerdo al anuario de estadísticas vitales del INEC año 2016.

Encuesta: Mediante una encuesta de 6 preguntas de las cuales las tres primeras eran de respuesta dicotómica, y las tres últimas de respuesta múltiple se evaluó la necesidad de la implementación y la viabilidad de la especialidad en cuidados paliativos. Esta encuesta se aplicó a 100 profesionales de la salud, los cuales incluían médicos especialistas, postgradistas y licenciadas en enfermería de diversos hospitales de la ciudad de Guayaquil.

\section{Resultados}

Guayaquil y Quito son las dos ciudades del Ecuador con mayor densidad poblacional, y en las cuales se concentran la mayor parte de los servicios de salud; en Guayaquil la tasa de incidencia de cáncer en hombres es de 142.1 y mujeres 165.5, mientras que en Quito en hombres es 228.1 por cada 100 mil habitantes, y 230.1 en mujeres por cada 100 mil.

En el gráfico 1, se observa la tendencia de la incidencia de cáncer que tiene un crecimiento exponencial y está proyectado al año 2020, en el cual la tasa de crecimiento es del $4 \%$ anual, así como, el uso del coeficiente de determinación $\left(\mathrm{R}^{2}\right)$, considerándose el $95 \%$ un parámetro adecuado de variabilidad que refleja la continuidad del comportamiento 
creciente de los cánceres, el cual sirve de pronóstico y toma de decisiones en el ámbito preventivo y paliativo.

Gráfico 1. Incidencia de cánceres. Hospital de SOLCA - Guayaquil. Proyección al 2020.

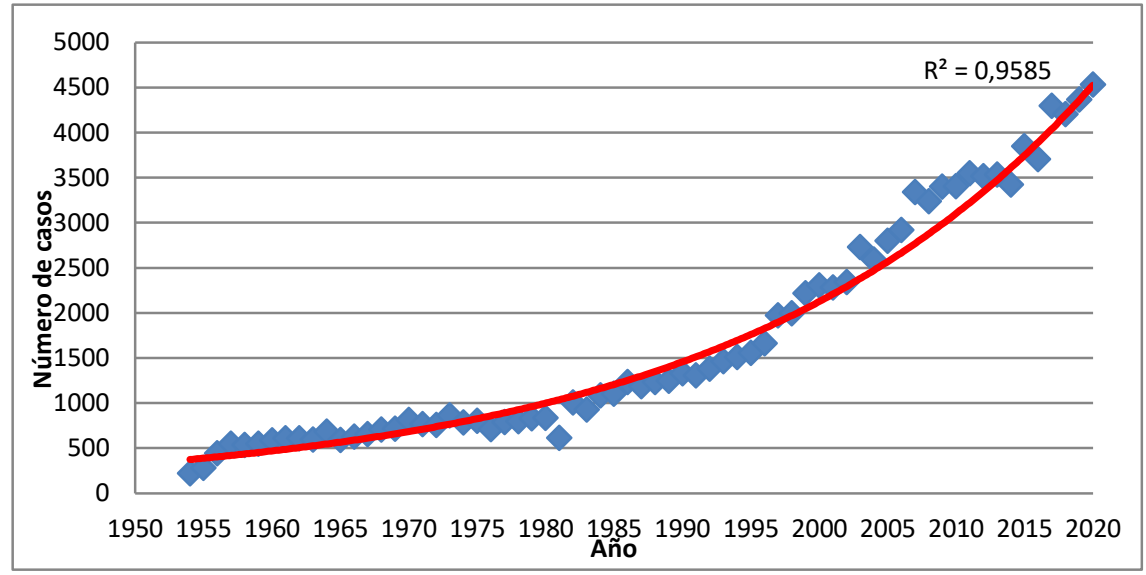

En el gráfico 2, se observa el comportamiento de la incidencia de los cánceres entre el 2011 al 2017, el mismo que con una tendencia polinomial y un coeficiente de determinación del $90 \%$ confirma la tendencia creciente de los casos.

Gráfico 2. Incidencia de cánceres. Hospital de SOLCA - Guayaquil. Período 2015 - 2017

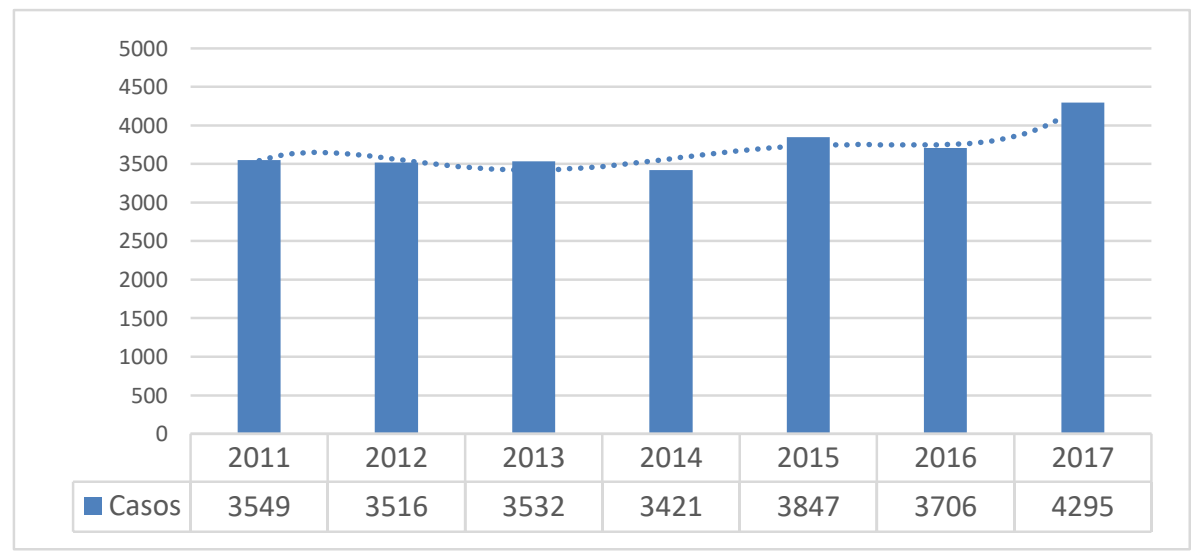

En la tabla 1, se observa que la proporción de casos de acuerdo al sexo es de $63 \%$ mujeres y $37 \%$ hombres, dicho porcentaje se mantiene en los últimos 3 años. En el gráfico 3, se observa que el mayor número de casos se encuentra a partir de los 45 años, siendo el grupo de 45 a 64 años el que ha tenido un incremento en los últimos 3 años, comparado con el resto de grupos etarios que se mantienen con un porcentaje de casos dentro de lo esperado. 
Tabla 1. Incidencia de Cánceres, según sexo. Hospital de SOLCA - Guayaquil. Período 2015 $-2017$

\begin{tabular}{|l|c|c|c|c|c|c|}
\hline & \multicolumn{2}{|c|}{2015} & \multicolumn{2}{c|}{2016} & \multicolumn{2}{c|}{2017} \\
\hline SEXO & No. & $\%$ & No. & $\%$ & No. & $\%$ \\
\hline Mujeres & 2430 & 63 & 2323 & 63 & 2708 & 63 \\
\hline Hombres & 1417 & 37 & 1383 & 37 & 1587 & 37 \\
\hline Total & 3847 & 100 & 3706 & 100 & 4295 & 100 \\
\hline
\end{tabular}

Gráfico 3. Incidencia de cáncer según grupo etario. Hospital de SOLCA - Guayaquil. Período $2015-2017$.

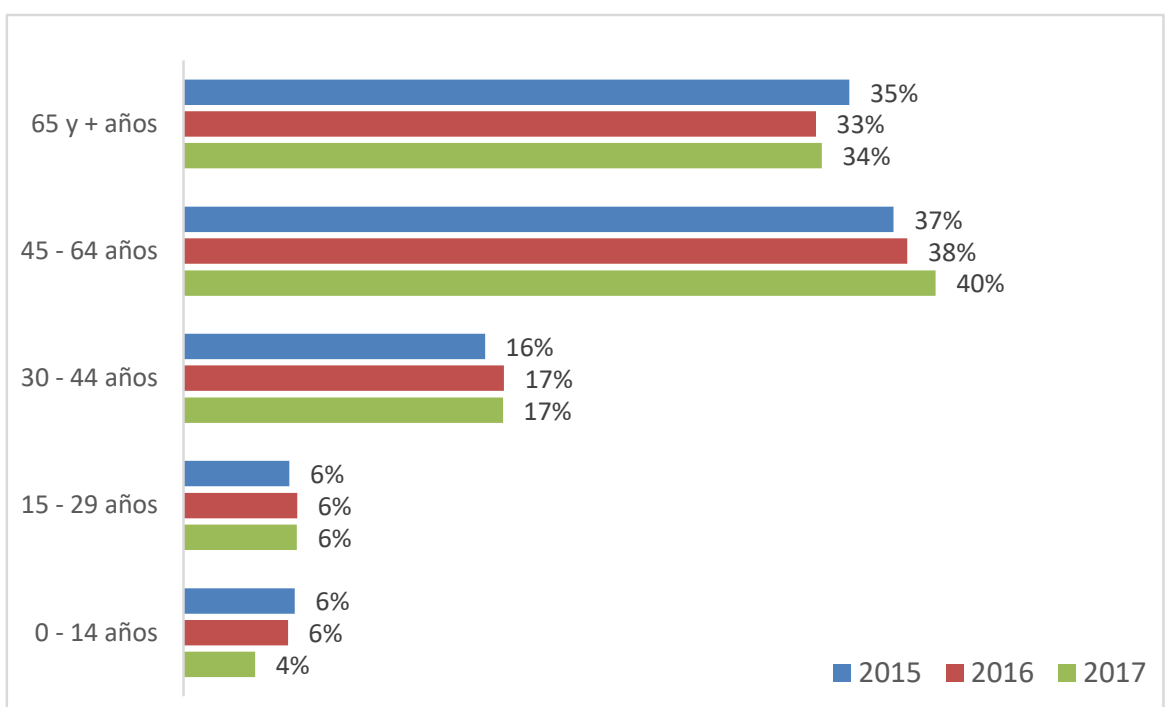

En la tabla 2, se observa que en las mujeres más del $40 \%$ de los casos están en el grupo de 45 a 64 años, mientras que en los hombres el mayor número de casos se encuentran en mayores de 65 años, y esta distribución se mantiene en los últimos 3 años.

En el gráfico 4, se observa que en mujeres el cáncer de mama ocupa el primer lugar, con una ligera disminución entre el 2015 al 2017, mientras que es de notoriedad que los cánceres de cérvix, tiroides y piel presentan un incremento continuo en los 3 últimos años. En cuanto a hombres el cáncer de próstata ocupa el primer lugar, y se debe considerar que el cáncer de colon - recto y estomago dentro del tracto digestivo, es una patología importante que mantiene un porcentaje considerable. 
Tabla 2. Incidencia de cáncer según sexo y grupo etario. Hospital de SOLCA Guayaquil. Período 2015 - 2017.

\begin{tabular}{|l|r|r|r|r|r|r|}
\cline { 2 - 8 } & \multicolumn{7}{|c|}{ HOMBRES } \\
\cline { 2 - 8 } & \multicolumn{2}{|c|}{2015} & \multicolumn{2}{c|}{$\mathbf{2 0 1 6}$} & \multicolumn{2}{|c|}{$\mathbf{2 0 1 7}$} \\
\hline GRUPO ETARIO & \multicolumn{1}{c|}{ No. } & \multicolumn{1}{c|}{ \% } & \multicolumn{1}{c|}{ No. } & \multicolumn{1}{c|}{ \% } & \multicolumn{1}{c|}{ No. } & \multicolumn{1}{c|}{$\%$} \\
\hline $0-14$ años & 115 & 8.12 & 109 & 7.88 & 99 & 6.24 \\
\hline $15-29$ años & 92 & 6.49 & 86 & 6.22 & 103 & 6.49 \\
\hline 30 - 44 años & 153 & 10.80 & 135 & 9.76 & 155 & 9.77 \\
\hline $45-64$ años & 406 & 28.65 & 446 & 32.25 & 529 & 33.33 \\
\hline $65 y+$ años & 651 & 45.94 & 607 & 43.89 & 701 & 44.17 \\
\hline Total & 1417 & 100.00 & 1383 & 100.00 & 1587 & 100.00 \\
\hline
\end{tabular}

\begin{tabular}{|c|c|c|c|c|c|c|}
\hline \multirow[b]{3}{*}{ GRUPO ETARIO } & \multicolumn{6}{|c|}{ MUJERES } \\
\hline & \multicolumn{2}{|c|}{2015} & \multicolumn{2}{|l|}{2016} & \multicolumn{2}{|c|}{2017} \\
\hline & \begin{tabular}{l|} 
No. \\
\end{tabular} & $\%$ & No. & $\%$ & \begin{tabular}{l|l} 
No. & \\
\end{tabular} & $\%$ \\
\hline 0 - 14 años & 111 & 4.57 & 95 & 4.09 & 63 & 2.33 \\
\hline $15-29$ años & 123 & 5.06 & 136 & 5.85 & 154 & 5.69 \\
\hline 30 - 44 años & 459 & 18.89 & 492 & 21.18 & 569 & 21.01 \\
\hline 45 - 64 años & 1036 & 42.63 & 970 & 41.76 & 1176 & 43.43 \\
\hline $65 y+$ años & 701 & 28.85 & 630 & 27.12 & 746 & 27.55 \\
\hline Total & 2430 & 100.00 & 2323 & 100.00 & 2708 & 100.00 \\
\hline
\end{tabular}

Gráfico 4. Incidencia según tipo de cáncer y sexo. Hospital de SOLCA - Guayaquil. Período $2015-2017$.

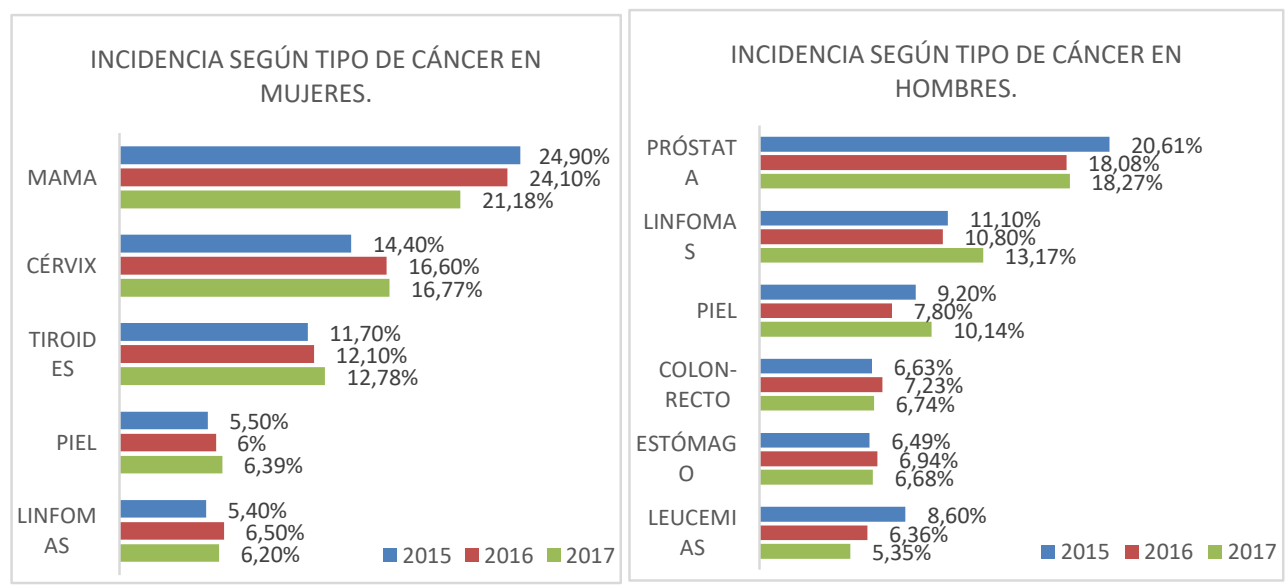

\section{Análisis de la Demanda de los cuidados paliativos como carrera Universitaria}

En la encuesta en la pregunta uno, 100 encuestados (100\%) de los encuestados consideró muy necesaria la especialidad de cuidados paliativos. La pregunta dos que analiza la oferta académica, el $93 \%$ desconoce si se oferta la especialidad, mientras que el $7 \%$ relaciona la existencia de ésta especialidad con la materia opcional que existe en otras especialidades médicas, pero no consta dentro del pensum académico. En la pregunta tres el $100 \%$ respondió que las universidades deberían ofertar esta especialidad. La pregunta cuatro, de los 9 temas que se deben abordar en la especialidad de cuidados paliativos, el tratamiento del dolor tema fue considerado el más relevante por el $100 \%$ de los encuestados. La pregunta cinco, la Universidad Católica de Santiago de Guayaquil fue elegida por el $86 \%$ de los encuestados y en la última pregunta con respecto a las unidades asistenciales donde debería desarrollarse la especialización, el 100 \% indicó que en SOLCA. 


\section{Discusión}

Las enfermedades crónicas se encuentran entre las primeras causas de muerte en el país, tanto en mujeres como en hombres. En la población femenina, en primer lugar se encuentran las enfermedades cardiovasculares como la hipertensión arterial e isquemia cardíaca, seguidas de diabetes, cerebrovasculares y neumonía. Mientras que en hombres las enfermedades crónicas no trasmisibles comparten los primeros lugares con las agresiones y los accidentes de tránsito.

Las enfermedades crónicas no trasmisibles son evitables si se trabaja de manera multisectorial en la promoción de la salud, en la prevención de la enfermedad y en el diagnóstico y tratamiento oportunos (MSP, 2010), en virtud de estos reportes de incremento de la ENT, es importante proyectarse a la atención paliativa desde las fases de diagnóstico lo cual permitirá un cambio real de la calidad de vida.

Recordando que la incidencia de cáncer es la línea de base para la investigación; así como, la prevención y control de los cánceres, se observa que el cáncer de mama se mantiene en el primer lugar en las mujeres y el de próstata en los varones; sin embargo, se evidencia un incremento en los casos de cáncer de tiroides y de piel en adultos.

Es de considerar que la actual supervivencia de los canceres de mama en mujeres y próstata en varones se ha incrementado [15], lo cual lleva a una mayor carga de esta enfermedad en el servicio de salud, requiriendo estos pacientes consultas para tratamiento de dolor y otros síntomas de tipo social, emocional y espiritual. Expectativa de crecimiento del país desde la mirada de organismos, como es la OMS y el INEC.

De acuerdo al resultado de la herramienta, se estimó que el $60 \%$ de los pacientes con enfermedades crónicas se beneficiarían de cuidados paliativos, esto incluye a los cuidadores inmediatos.

El grupo A que representa el $60-70 \%$ y genera un sufrimiento leve requerirá de atención paliativa por un facultativo que posea conocimientos paliativos para que pueda ofrecer una buena información. En relación al grupo B que revela un sufrimiento moderado 25-30 $\%$ necesitará a un profesional con mejores conocimientos en cuidados paliativos para un mejor control de síntomas. El grupo C que es del 5-10\% que son pacientes con situación complicada que generan un sufrimiento intenso necesitara de un especialista en cuidados paliativos para mejorar la cálida de vida disminuyendo el sufrimiento del paciente y su familia.

Con estos resultados, es importante que teniendo en el País una normativa a nivel Gubernamental y contando el MSP con una guía práctica clínica para una toma de decisiones dirigidas, las universidades se comprometan a formar especialistas en cuidados paliativos para brindar mejor calidad de vida y tengan siempre el acceso a los niveles apropiados de atención para pacientes con una enfermedad que limita la vida. 
Necesidad estimada de Cuidado Paliativo $®$

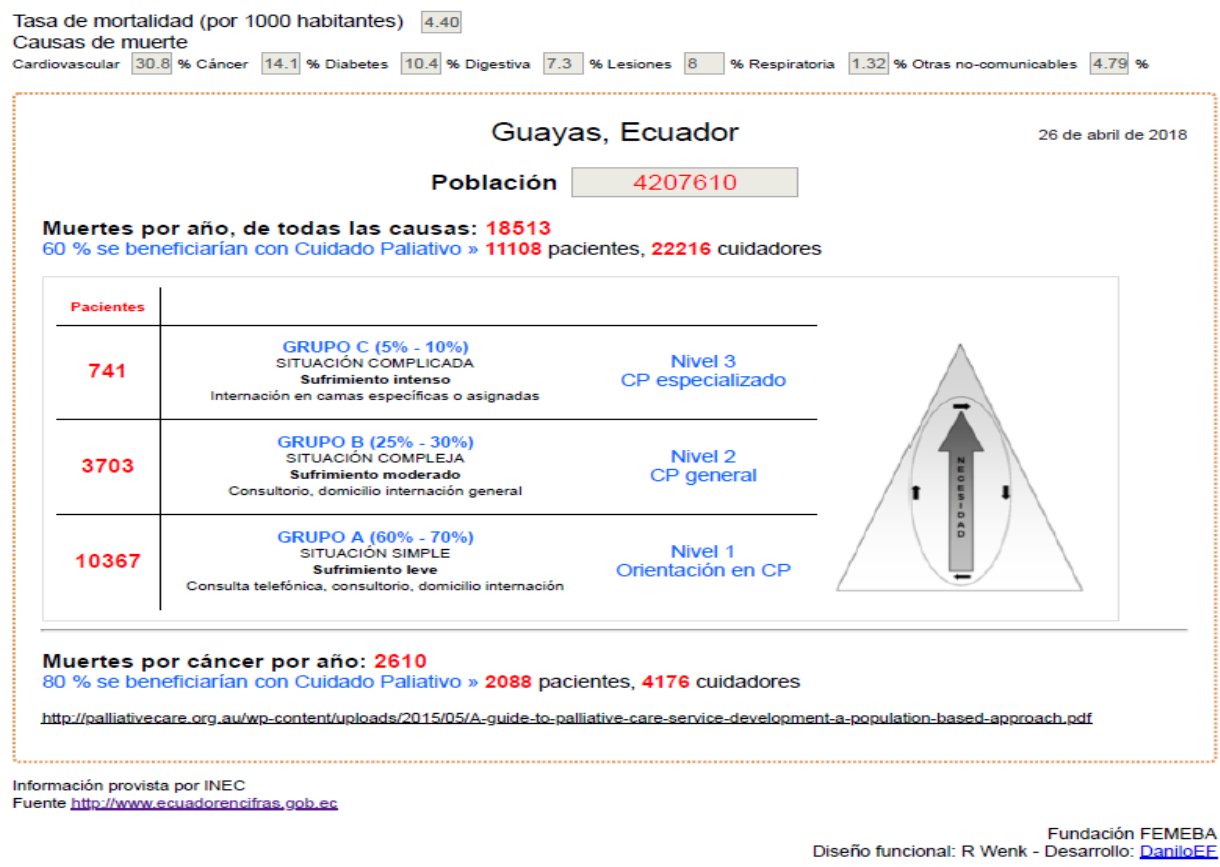

Del análisis de pertinencia de la carrera del área de cuidados paliativos, y de acuerdo al PNBV, mejorar la calidad de vida de la población se considera un reto amplio que demanda la consolidación de los logros alcanzados en los últimos años, mediante el fortalecimiento de políticas intersectoriales y la consolidación del Sistema Nacional de Inclusión y Equidad Social.

Mejorar la calidad de vida de la población demanda la universalización de derechos mediante la garantía de servicios de calidad, lo cual debe ir conectado con la calidad y la calidez en los servicios de atención con personal sanitario capacitado formalmente. Es importante consolidar políticas de prevención y paliación en todas las etapas de las enfermedades y no solo en la última fase de la vida o de la enfermedad. De acuerdo a los resultados de la encuesta aplicada, se evidenció el desconocimiento sobre la importancia que tiene en los pacientes el manejo de los aspectos emocionales, sociales y espirituales que también son parte importante del tratamiento integral del paciente. Así también.

El incremento en la expectativa de vida de los ecuatorianos junto a la transición demográfica, que de acuerdo al INEC se dio a partir del año 2012, es decir ha disminuido la tasa de natalidad y la población está envejeciendo, hace imperativa la necesidad de esta especialización con personal formado para brindar calidad de vida y calidad de partida, ya que en los próximos años aumentará la población de personas adultas, y con el estilo de vida actual, los factores de riesgo ambientales, entre otros, se espera que se incrementen las enfermedades crónicas no transmisibles.

Una vez realizado este estudio, desde la nueva visión que plantea el PNBV que promueve el fortalecimiento del talento humano y la calidad educativa como principios claves, para brindar calidad de vida hasta el último momento de su existencia, este proyecto de especialización en cuidados paliativos genera una expectativa relevante para brindar calidad de atención a este grupo poblacional en todos los niveles de salud, incluyendo a sus cuidadores. 
Incluir la cátedra de cuidados paliativos de manera formal, especialmente en las carreras de medicina y enfermería, tanto en pregrado como en postgrado. Con todos los antecedentes señalados, urge contar con programas de posgrado en el área de la salud para la formación de cuarto nivel de Especialistas en Cuidados Paliativos [16].

\section{Conclusiones}

Luego de la revisión y análisis de las respuestas proporcionadas por los profesionales de la salud, se infiere que es sumamente importante contar con programas de posgrado para iniciar la formación de especialistas en Cuidados Paliativos debido a que es un personal profesional estratégico, para elevar los estándares de calidad de vida de la población guayaquileña y ecuatoriana; así mismo, contribuir a reducir la brecha actualmente existente en el Ecuador. Revisando el Atlas de Cuidados paliativos en Latinoamérica, edición cartográfica 2013, Ecuador se ubica en el grupo 2, equivalente a "Desarrollo insipiente", "actividad desconocida". Ecuador no registra atención profesional paliativista; y las Facultades de Ciencias Médicas: sin registro de programas.

\section{Agradecimientos}

Se reconoce a las personas que participaron indirectamente en el estudio tales como el personal técnico y otras en general del Instituto Oncológico Nacional "Dr. Juan Tanca Marengo", Solca- Guayaquil.

\section{Información adicional}

\section{Abreviaturas}

ALCP: Asociación Latinoamericana de cuidados paliativos.

CEAACES: Consejo de Evaluación, Acreditación y Aseguramiento de la Calidad de la Educación Superior.

CP: Cuidados Paliativos.

INEC: Inatituto Nacional de Estadísticas y Censos.

ENT: enfermedades no transmisibles.

EPOC: enfermedad pulmonar obstructiva crónica.

MSP: Ministerio de Salud Pública.

OMS: Organización mundial de la Salud.

PNBV: Plan Nacional del Buen Vivir.

Solca: Sociedad de Lucha contra el cáncer.

SIDA: Síndrome de Inmunodeficiencia Adquirida.

UCSG: Universidad Católica de Santiago de Guayaquil.

UVPN: Valor Predictivo Negativo.

Nota del Editor

La Revista Oncología Ecu

permanece neutral con

respecto a los reclamos

jurisdiccionales en mapas

publicados y afiliaciones 
Archivos Adicionales

Ninguno declarado por los autores.

Fondos

Los fondos de la investigación fueron propios de los autores del presente artículo.

\section{Disponibilidad de datos y materiales}

Existe la disponibilidad de datos bajo solicitud al autor de correspondencia. No se reportan otros materiales.

\section{Contribuciones de los autores}

$\mathrm{MV}, \mathrm{NL}$, realizaron la idea de investigación, revisión bibliográfica, el análisis crítico del artículo. $\mathrm{RQ}$ realizó la recolección de datos, análisis estadístico, escritura del artículo. MV realizó las correcciones. Todos los autores leyeron y aprobaron la versión final del artículo.

Aprobación de ética y consentimiento para participar

No aplica ya que es un estudio de bases de datos.

\section{Consentimiento para publicación}

No aplica.

\section{Información de los autores}

Mariana Vallejo Martínez, Médica tratante del Servicio de Dolor y Paliativos del Instituto Oncológico Nacional "Dr. Juan Tanca Marengo".-Solca- Guayaquil. Master en Atención paliativa.

\section{iD https://orcid.org/0000-0001-5727-262X}

Nancy Lino, Jefe Servicio de Cuidados Paliativos y Manejo del Dolor del Instituto Oncológico Nacional "Dr. Juan Tanca Marengo".Solca- Guayaquil.

Rina Quinto, departamento de estadísticas del Instituto Oncológico Nacional "Dr. Juan Tanca Marengo".Solca- Guayaquil. 
Revisiones por pares

Acceda a la revisión de pares académicos en el siguiente enlace: https://publons.com/review/3

Abreviaturas en la referencias

DOI: Digital Object

Identifier

PMID: PubMed Identifier

SU: Short URL

\section{Referencias}

1. Doyle D, Hanks G, Cherny N, Calman K. Oxford Textbook of Palliative Medicine. J R Soc Med. 2004;97(7):356-357. PMCID: PMC1079539.

2. Walsh D. Medicina Paliativa. Elsevier Texbook. Madrid 2010. ISBN: 9788480868181

3. Organización Mundial de la salud. [intenet]. Actualización 2017. Cáncer: Cuidados Paliativos. Consultado 2016.01.03. SU: goo.gl/VyE5S1

4. Ministerio de Salud Pública del Ecuador. [internet]. Actualización 2017. Cuidados paliativos, Guía de Práctica Clínica. Quito: MSP; 2014. SU: goo.gl/nopmEh

5. Ministerio de Salud Pública, Plan Nacional de Cuidados Paliativos. Subsecretaría Nacional de Gobernanza de la Salud, Dirección Nacional de Políticas y Modelamiento del Sistema Nacional de Salud, Dirección Nacional de Normatización, 2015, Quito, Ecuador. SU: goo.gl/Sbvszz

6. Ministerio de Salud Pública del Ecuador. [internet]. Actualización 2017. Guía de Cuidados paliativos para el Ciudadano. Quito: MSP; 2014. SU: goo.gl/7PCdmv

7. Morin E. Epistemologia de la complejidad. En: D. Fried Schnitman. Nuevos paradigmas, cultura y subjetividad. Buenos Aires: Paidós, 1994;421-442.

8. Larrea E. El currículo de la educación superior desde la complejidad sistémica. Curso: Estudio de pertinencia para las carreras universitaria, CES, Salinas (2014). SU: goo.gl/BBy7Lj

9. CEAACES. Modelo genérico de evaluación del entorno de aprendizaje de carreras presenciales y semipresenciales de las universidades y escuelas politécnicas del Ecuador. Versión 2. Quito 2015.SU: goo.gl/AYkxwi

10. SENPLADES. Plan Nacional del Buen vivir Volumen 8 - Número 15, Septiembre 2015, pp. 69 -82 Vivir 2013 - 2017. 2014. SU: goo.gl/5SsnQt

11. SENPLADES. Plan Nacional del Buen Vivir 2013 - 2017. Estrategia Territorial. Agendas Zonales. 2014. SU: goo.gl/yf3q4T

12. INEC. [intenet]. Actualización 2017. Ecuador en cifras de salud. Consultado 2016.01.03. SU: goo.gl/aSCRu9

13. Asociación Latinoamericana de Cuidados Paliativos. [internet]. Actualización 2017. Avances en Cuidados paliativos. Consultado 2016.01.03. SU: goo.gl/E9igZ7

14. WHO. [internet]. Actualización 2017. Cancer Incidence in Five Continents.. Consultado 2016.01.03. SU: ci5.iarc.fr

15. Allemani C, Matsuda T, Di Carlo V, Harewood R, Matz M, Nikšić M, et al. CONCORD Working Group. Global surveillance of trends in cancer survival 2000-14 (CONCORD-3): analysis of individual records for 37513025 patients diagnosed with one of 18 cancers from 322 population-based registries in 71 countries. Lancet. 2018 Mar 17;391(10125):1023-1075. DOI: 10.1016/S0140-6736(17)33326-3.

16. Pastrana T, De lima L, Pons JJ, Centeno C. Atlas de Cuidados Paliativos en Latinoamérica. Asociación Latinoamericana en Cuidados Paliativos. SU: goo.gl/j99URx 